\title{
Effectiveness of a Laboratory Gate-Keeping Strategy to Overcome Inappropriate Test Utilization for the Diagnosis of Heparin-Induced Thrombocytopenia
}

\author{
Janne Cadamuro, MD ${ }^{1}$ Cornelia Mrazek, MD ${ }^{1}$ Helmut Wiedemann, MD ${ }^{1}$ Thomas Klaus Felder, PhD ${ }^{1}$ \\ Hannes Oberkofler, PhD ${ }^{1}$ Elisabeth Haschke-Becher, MD ${ }^{1}$ Giuseppe Lippi, MD²
}

\author{
${ }^{1}$ Department of Laboratory Medicine, Paracelsus Medical University, \\ Salzburg, Austria \\ 2 Section of Clinical Biochemistry, University Hospital of Verona, \\ Verona, Italy
}

Semin Thromb Hemost 2017;43:645-648

The purpose of medical laboratories is to provide analyses from blood and other specimens to aid diagnosis and treatment. The analytical spectrum provided by laboratories all over the world has expanded continuously over the past decades. The increasing availability of tests with fast turnaround times combined with defensive medicine strategies has led to an overuse of laboratory diagnostics. ${ }^{1-3}$ Health care expenditures, in turn, increase every year. In Austria, for example, respective per capita expenses related to healthcare increased from $\$ 2,249$ USD in 1995 to $\$ 5,038$ USD in 2014 (9.5-11.2\% of gross domestic product, respectively). ${ }^{4}$ Although in vitro diagnostic (IVD) expenses contribute only approximately $0.8 \%$ to these numbers, ${ }^{5}$ laboratories have to find strategies to overcome inappropriate test utilization, particularly because an overuse of laboratory diagnostics might even be harmful to patients. ${ }^{6}$ Such strategies include educational interventions, ${ }^{7}$ laboratory diagnostic pathways, ${ }^{8}$ minimum retesting intervals, ${ }^{9}$ and reimbursement or funding models amongst others. The model we describe here is a gatekeeping strategy. We chose laboratory diagnostics for detecting heparin-induced thrombocytopenia (HIT) type II, a prothrombotic immune response to heparin treatment, potentially leading to life-threatening conditions, ${ }^{10}$ whose diagnostic challenges have also been recently highlighted in this journal. ${ }^{11}$ Detection of anti-PF4/heparin antibodies (HIT$\mathrm{Ab})$ during the disease can be performed either using functional methods, such as platelet aggregation procedures or serotonin releasing tests, or using immunoassays. ${ }^{12,13}$ In the department of laboratory medicine of the Paracelsus Medical University (Salzburg, Austria), only the latter type of test is provided. This test was chosen for a gate-keeping strategy

published online July 27, 2017
Issue Theme Editorial Compilation IV; Guest Editors, Emmanuel J. Favaloro, PhD, FFSc (RCPA), and Giuseppe Lippi, MD. because respective test ordering is done quite often as compared with the much lower and predictable frequency of HIT, ranging from 0.3 to $3 \%$, depending on the patient collective and the type of heparin used. ${ }^{12,14}$ Prior to antibody testing, pretest probability can be estimated using a scoring system, commonly known as the 4Ts, consisting of four questions, each with three possible answers. Each answer reflects 0,1 , or 2 scoring points. A total of $\leq 3$ points is associated with a negative predictive value (NPV) of $99.8 \%$ for the presence of HIT regardless of the party responsible for scoring or prevalence of HIT. ${ }^{15}$ Based on this evidence, we implemented the respective questions into our physician order/entry system, providing answer options as separate dropdown menus. Answering all the questions was made mandatory prior to $\mathrm{HIT}-\mathrm{Ab}$ test ordering. The answers were automatically translated into the respective point values and summed. This information was then transferred into the laboratory information system (LIS). In test orders with a value $\leq 3$, the order was held up and the clinician was informed that the probability of a positive HIT-Ab test in the patient was $<2 \%$. The clinician was also asked to call the laboratory if he/she still wished the analysis to be performed regardless of the scoring value. Implementation was done in accordance with the interdisciplinary coagulation working group of the hospital, comprising representatives from five clinics, the department of laboratory medicine, and the pharmacy. Internal medicine coagulation consultative clinicians were directly informed about the date of implementation and all other departments were informed using the hospital intranet. Testing was performed using the ID-Particle Gel Immuno Assay (PaGIA) Heparin/PF4 Antibody Test (Bio-Rad Laboratories, Hercules, CA).
Copyright (c) 2017 by Thieme Medical Publishers, Inc., 333 Seventh Avenue, New York, NY 10001, USA. Tel: +1(212) 584-4662.
DOI https://doi.org/ 10.1055/s-0037-1604054. ISSN 0094-6176. 
Three months after implementation of this gate-keeping strategy, the results of 55 HIT-Ab test orders were evaluated ( $47.3 \%$ from intensive care units, $14.5 \%$ from oncology or radiotherapy, $9.1 \%$ from surgical departments and others). We aimed to answer the following questions: (1) Are 4Ts scorings reported too high, too low, or correctly? (2) How many orders could be saved by implementation of the HIT-4T score gate-keeper strategy? (3) How often are the held-up orders re-ordered and are the scores different between these orders in the same patient? We did not seek approval from the local ethics committee since the study was not experimental and undertaken retrospectively in the context of routine investigations and quality assurance. The study was conducted according to the Declaration of Helsinki.

Since most of the questions asked in the HIT-4T score rely on either clinical or therapeutic information, we could not check for correctness of answers to questions 2, 3, and 4 . However, the first question asks for proportional and absolute platelet count decrease and information available within the LIS. Therefore, to answer our first study question, we scored the respective platelet count measurements and compared them to the scores provided by the clinicians in patients who had a HIT-Ab test ordered. As the input of additional information by clinicians and/or nurses (e.g., indication, medication) into the order/entry system is often considered annoying and time consuming, it is sometimes bypassed by providing mean- ingless information (e.g., "xy" or “...”). We therefore expected the provided scores to be higher than the respective true values in some or perhaps many cases so that the clinician receives HIT-Ab analyses regardless of true scoring information. Fortunately, scoring of question 1 was completed correctly in $47.3 \%$ of cases, whereas in $45.4 \%$ of cases, scores were too low, leaving only $7.3 \%$ of cases where the question was scored too high (-Fig. 1).

Evaluation of the data regarding our second and third study question showed that $52.7 \%$ of orders $(n=29)$ were held up, and in only three cases did the clinician call to request sample analysis despite the fact that the $4 \mathrm{~T}$ score totaled $\leq 3$ in these patients. In seven cases $(26.9 \%$ of the remaining held-up orders), an electronic re-order was placed for HIT-Ab testing. The respective total HIT-4T score in these re-orders was higher, identical, and lower in 2, 3, and 2 cases, respectively. In the remaining $73.1 \%$ of held-up orders, no retest was ordered, leaving the clinician apparently satisfied with the information provided on the NPV.

This study confirms that information entered for the $4 \mathrm{~T}$ score is mostly done correctly, without trying to bypass the system. Depending on the HIT-Ab assay in use and based on the number of respective orders sent to our coagulation laboratory, the implementation of the HIT-4T score gatekeeper strategy may have resulted in test-performance savings ranging from approximately $1,500 €$ to $4,500 €$ per year

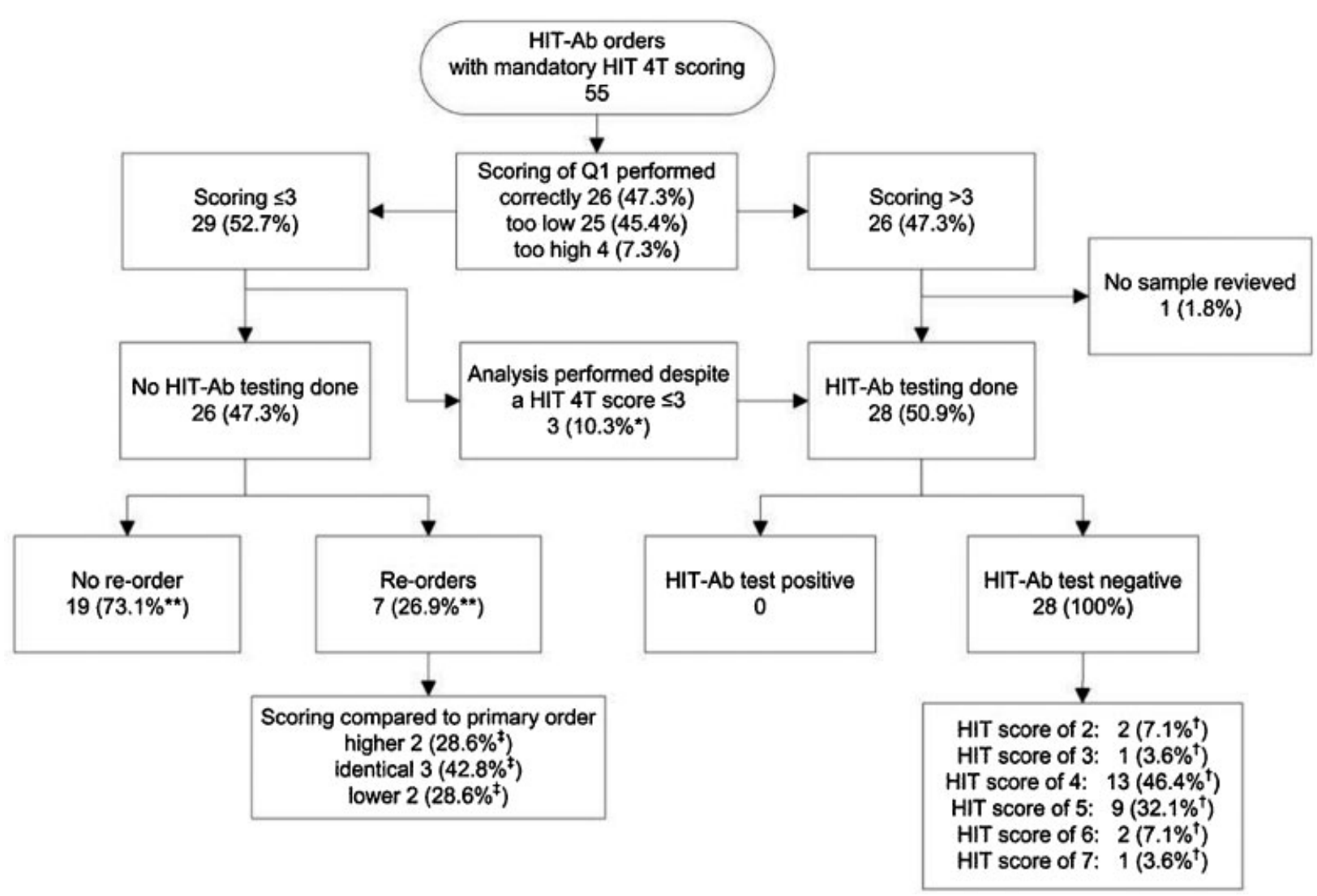

Fig. 1 Results from the implementation of HIT-4T scoring as mandatory step prior to HIT-Ab testing. HIT-Ab-anti-PF4/heparin antibodies; Q1, Question 1 of the HIT-4Ts score (proportional and absolute platelet count decrease); " $n$ " represents number; *amount per orders held up; **amount per orders where no HIT-Ab testing was performed; ${ }^{\dagger}$ amount per samples tested negative; ${ }^{\dagger}$ amount per re-orders. 
on the condition that the test was available on a daily basis and only reagent costs were calculated. Implementation of the mandatory $4 \mathrm{~T}$ scoring was very well accepted, since no complaints were received. Of further interest, even in the samples eventually tested for HIT-Ab, all (28/28) were identified to be negative, thereby confirming the low "hit-rate" of positive tests and despite a proportion of these patients having high HIT-4T scores (-Fig. 1).

Under-diagnosis of HIT is potentially life-threatening, but also an over diagnosis of this condition may have serious clinical consequences; thus, an efficient diagnostic approach designed to improve appropriateness is crucial. ${ }^{16}$ According to the results of our investigation, we can conclude that implementation of the HIT-4Ts scoring into a physician's order/entry system provides a useful gate-keeping tool for reducing the number of laboratory tests. It does not jeopardize the quality of patient care, may be associated with significant savings, and is very well received by clinicians. Accordingly, we consider this to be a mandatory step prior to HIT-Ab diagnostics for modern laboratory systems.

\section{References}

1 Carter R. Report of the Second Phase of the Independent Review of NHS Pathology Services in England. United Kingdom: Department of Health; 2008

2 Studdert DM, Mello MM, Sage WM, et al. Defensive medicine among high-risk specialist physicians in a volatile malpractice environment. JAMA 2005;293(21):2609-2617

3 Zhi M, Ding EL, Theisen-Toupal J, Whelan J, Arnaout R. The landscape of inappropriate laboratory testing: a 15-year metaanalysis. PLoS One 2013;8(11):e78962
4 The World Bank Group. Health expenditure. Available at: http://data.worldbank.org/indicator/SH.XPD.TOTL.ZS. Accessed April 21, 2017

5 European Diagnostic Manufacturers Association (EDMA). European IVD Market Statistics Report 2011-2015

6 Freedman DB. Towards better test utilization-strategies to improve physician ordering and their impact on patient outcomes. EJIFCC 2015;26(01):15-30

7 Wertheim BM, Aguirre AJ, Bhattacharyya RP, et al. An educational and administrative intervention to promote rational laboratory test ordering on an academic general medicine service. Am J Med 2017;130(01):47-53

8 Hoffmann GE, Aufenanger J, Födinger M, et al. Benefits and limitations of laboratory diagnostic pathways. Diagnosis 2014;1 (04):269-276

9 Lang T, Croal B. National minimum retesting intervals in pathology. London: The Royal College of Pathologists; 2015. Contract no: G147

10 Prechel M, Walenga JM. Heparin-induced thrombocytopenia: an update. Semin Thromb Hemost 2012;38(05):483-496

11 Berroëta C, Crespin M, Bouabdallah K, et al. Diagnostic performance of a new rapid lateral flow immunoassay in patients suspected of heparin-induced thrombocytopenia and its clinical consequences. Semin Thromb Hemost 2016;42(01):69-74

12 Greinacher A. CLINICAL PRACTICE. Heparin-induced thrombocytopenia. N Engl J Med 2015;373(03):252-261

13 Favaloro EJ, McCaughan G, Pasalic L. Clinical and laboratory diagnosis of heparin induced thrombocytopenia: an update. Pathology 2017;49(04):346-355

14 Warkentin TE. Heparin-induced thrombocytopenia in critically ill patients. Semin Thromb Hemost 2015;41(01):49-60

15 Cuker A, Gimotty PA, Crowther MA, Warkentin TE. Predictive value of the 4Ts scoring system for heparin-induced thrombocytopenia: a systematic review and meta-analysis. Blood 2012;120 (20):4160-4167

16 Favaloro EJ. Toward improved diagnosis of HIT. Blood 2015; 126(05):563-564 